\title{
Myocardin and Stat3 act synergistically to inhibit cardiomyocyte apoptosis
}

\author{
Yuan Xiang ${ }^{1, *}$, Xing-Hua Liao ${ }^{1, *}$, Jia-Peng Li $^{1}$, Hui Li ${ }^{1}$, Huan Qin ${ }^{1}$, Ao Yao ${ }^{1}$, Cheng-Xi \\ $\mathrm{Yu}^{1}$, Peng $\mathrm{Hu}^{1}$, Wei Guo ${ }^{3}$, Chao-Jiang Gu${ }^{2}$ and Tong-Cun Zhang ${ }^{1,2}$ \\ ${ }^{1}$ Institute of Biology and Medicine, Wuhan University of Science and Technology, Hubei, 430081, P.R. China \\ ${ }^{2}$ Key Laboratory of Industrial Fermentation Microbiology, Ministry of Education and Tianjin, College of Biotechnology, Tianjin \\ University of Science and Technology, Tianjin, 300457, P.R. China \\ ${ }^{3}$ Shenzhen Ritzcon Biological Technology Co., LTD, Shenzhen, Guangdong, 518000, P.R. China \\ *These authors have contributed equally to this work \\ Correspondence to: Xing-Hua Liao, email: xinghualiao@hotmail.com \\ Tong-Cun Zhang, email: zhangtongcun@wust.edu.cn \\ Chao-Jiang Gu, email: guchaojiang@wust.edu.cn
}

Keywords: myocardial; Stat3; cardiomyocyte apoptosis

Received: May 10, $2017 \quad$ Accepted: July 26, $2017 \quad$ Published: August 24, 2017

Copyright: Xiang et al. This is an open-access article distributed under the terms of the Creative Commons Attribution License 3.0 (CC BY 3.0), which permits unrestricted use, distribution, and reproduction in any medium, provided the original author and source are credited.

\section{ABSTRACT}

Signal transducer and activator of transcription 3 (Stat3) and Myocardin regulate cardiomyocyte differentiation, proliferation, and apoptosis. We report a novel aspect of the cellular function of Myocardin and Stat3 in the regulation of cardiomyocyte apoptosis. Myocardin and Stat3 showed anti-apoptotic function by increasing the expression of $\mathrm{Bcl}-2$ while reducing expression of the pro-apoptotic genes Bax, Apaf1, caspase-9, and caspase-3. Moreover, myocardin/Stat3-mediated activation of Bcl-2 and Mcl-1 transcription is contingent on the CArG box. Myocardin and Stat3 synergistically inhibited staurosporine-induced cardiomyocyte apoptosis by upregulating expression of anti-apoptotic $\mathrm{Bcl}-2$ and $\mathrm{Mcl}-1$ in neonatal rat cardiomyocytes. These results describe a novel anti-apoptotic Myocardin/Stat3 signaling pathway operating during cardiomyocyte apoptosis. This provides a molecular explanation for cardiomyocyte apoptosis inhibition as a critical component of myocardial protection.

\section{INTRODUCTION}

Cardiomyocyte apoptosis is a key turning point in the cell death process [1-3], and critical in the pathogenesis of various cardiovascular diseases. Apoptosis inhibition is an important target for therapeutic intervention. However, the mechanism of cardiomyocyte apoptosis is not well understood.

Signal transducer and activator of transcription 3 (Stat3) is a kind of transcription which mediated cell growth, differentiation, and survival of various cell types [4-7]. Stat3 activation results in the upregulation of various genes involved in cell survival and proliferation, such as those encoding Bcl-2, Bcl-XL, Mcl-1 (myeloid cell leukemia-1), cyclin-D1 and c-Myc [8-10]. Inhibition of Stat 3 activity reduced the expression of survivin in primary effusion lymphoma-induced cell apoptosis [11]. Importantly, constitutive cardiomyocyte- restricted deletion of Stat3 results in increased apoptosis $[12,13]$. In this study, we demonstrate that Stat3 and Myocardin directly interact in vivo, suggesting that these two transcription factors synergistically enhance the antiapoptosis effect in cardiomyocytes.

Myocardin is highly expressed in embryonic cardiac and smooth muscle [14-16], acting as a potent transcriptional activator that activates CArG boxdependent cardiac promoters by forming a ternary complex with SRF [17-19]. Previous studies have shown that SRF protects cells from apoptotic cell death by regulating anti-apoptosis gene Bcl-2 and Mcl-1 activity 
$[20,21]$. Myocardin inhibits the cell-cycle progression at the $\mathrm{G} 2 / \mathrm{M}$ phase [22], influences failing heart gene expression and function [23, 24], and Myocardin loss in cardiomyocytes triggers programmed cell death [25]. However, the molecular mechanism of Myocardin in cardiomyocyte apoptosis mediated-heart failure remains unknown.

We hypothesized that Myocardin and Stat3 have a synergistic anti-apoptosis effect in cardiomyocytes. Herein we demonstrate an interaction between Myocardin and Stat3 to inhibit apoptosis and upregulate anti-apoptotic gene expression for myocardial protection through cardiomyocyte apoptosis. We define a new role for Myocardin and Stat3 as a transcriptional repressor and inhibitor of cardiomyocyte apoptosis.

\section{RESULTS}

\section{Myocardin and Stat 3 synergistically inhibit staurosporine-induced cardiomyocyte apoptosis}

Both myocardin and Stat3 can mediate cardiomyocyte growth, differentiation, and survival, so we determined the role of Myocardin and Stat3 in cardiomyocyte apoptosis. The TUNEL assay results for Myocardin transfected cardiomyocyte cells indicated a decrease of nearly $50 \%$ in TUNEL-positive cells in staurosporine-treated cells as compared to the controls, while Stat 3 transfected cardiomyocyte cells indicated a decrease of almost 55\% (Figure 1A and 1B). Moreover, Myocardin and Stat 3 cotransfected cardiomyocyte cells indicated an approximately $70 \%$ decrease (Figure 1A and 1B). Very few TUNEL-positive cells were present in the control cells. Importantly, the Annexin V-FITC apoptosis detection assay data quantify early and late events in the course of apoptosis. The Annexin V-FITC results show that Myocardin significantly diminished the number of apoptotic cells induced by staurosporine (Figure 1C). Similarly, Stat3 significantly reduced the apoptosis rate (Figure 1C). The observed number of apoptotic cells cotransfected with Myocardin and Stat3 declined (Figure 1C). These data suggest that cooperativity of Myocardin and Stat3 inhibits staurosporine-induced cardiomyocyte apoptosis.

\section{Myocardin and Stat3 enhance the expression of anti-apoptotic genes}

The expression of anti-apoptotic markers was examined by RT-PCR and western blot to demonstrate the effect of Myocardin and Stat3 on regulating the expression of the anti-apoptotic markers (Bcl-2 and Mcl-1) in the presence of staurosporine. Our data showed that both Myocardin and Stat 3 mitigate the effect of staurosporine on the expression of anti-apoptotic genes. The expression of Bcl-2 and Mcl-1 was enhanced after transfection with Myocardin and dramatically upregulated when transfected with the two proteins (Figure 2A). Further expression changes were also measured on the protein level by western blotting, and enhanced expression of Bcl-2 and Mcl-1 was observed when transfected with Myocardin and Stat3 (Figure 2B). As shown in Figure 2C and 2D, Myocardin and Stat3 strongly amplified the expression of Bcl-2 and Mcl-1. Bcl-2 and Mcl-1 protein expression was increased in cardiomyocyte cells transfected with Myocardin, Stat3, and Myocardin/Stat 3 compared to the cells transfected with the pcDNA3.1 vector by immunofluorescence staining. Notably, Bcl-2 and Mcl-1 were located in the cytoplasm when transfected with vector but accumulated around the nuclei in cells cotransfected with Myocardin and Stat3. These observations suggest that Myocardin and Stat3 synergistically enhance the expression of anti-apoptotic genes to inhibit staurosporine-induced cardiomyocyte apoptosis. Our results indicate that Myocardin and Stat3 play a pivotal role as an antiapoptotic factor in cardiomyocyte cells, and targeting Myocardin and Stat 3 activity led to the induction of antiapoptosis activity.

\section{Myocardin and Stat3 mediated anti-apoptosis by the intrinsic or mitochondrial apoptotic pathway}

It has been reported that the role of Bcl-2 family of proteins was evaluated by the measurement of the antiapoptotic protein $\mathrm{Bcl}-2$ and the pro-apoptotic protein Bax [26-29]. The expression of Bcl-2 was increased in Myocardin and Stat3 transfected cardiomyocytes (Figure $3 \mathrm{~A}$ and $3 \mathrm{~B}$ ). Bax expression was decreased in the transfected Myocardin and Stat3 cardiomyocytes compared to the control cardiomyocytes (Figure 3A and $3 \mathrm{~B})$.

The functional significance of these expression patterns is derived from the potential for heterodimer formation between Bax and Bcl-2. A high Bax:Bcl-2 ratio enhances the probability for Bax homodimer formation and cell death signaling, whereas a relative abundance of Bcl-2 favors formation of Bcl-2/Bax heterodimers and Bcl-2/Bcl-2 homodimers, which promote cell survival $[30,31]$. We investigated the mechanism of Myocardin and Stat 3 enhancement of anti-apoptotic gene expression. We calculated the ratio of $\mathrm{Bcl}-2$ expression to $\mathrm{Bax}$ expression, and the ratio was reversed in cardiomyocytes (elevated Bcl-2 levels and transient reduction of Bax levels). The ratio of Bcl-2:Bax indicated that when the ratio was more than 0.5 , an apoptotic procedure switches on. The densitometric analysis showed a high Bcl-2/Bax ratio in transfected Myocardin and Stat 3 cardiomyocytes (Figure 3C and 3D), which suggests that Myocardin and Stat3 have an anti-apoptotic function by increasing the expression of Bcl-2 while reducing the pro-apoptotic gene-Bax. 
Because a low Bcl-2/Bax ratio enhances cell death signaling, we next determined apoptotic protease activating factor-1 (Apaf-1), the initiator caspase-9, and the effector caspase- 3 expression. The cleavage of caspases is aided by the release of caspase-activating factors, particularly cytochrome $\mathrm{C}$ from the mitochondrial membrane into the cytosol $[32,33]$. Once released into the cytosol, cytochrome C activates pro-caspase 9 [34]. Caspase- 3 is considered the most important of the executioner caspases and is activated by the initiator caspase-9. Our results show that transfected Myocardin and Stat 3 cardiomyocytes significantly reduce the release of cytochrome $\mathrm{C}$ and the expression of Apaf-1, caspase-9, and caspase- 3 as compared to the controls (Figure $3 \mathrm{E}$ and

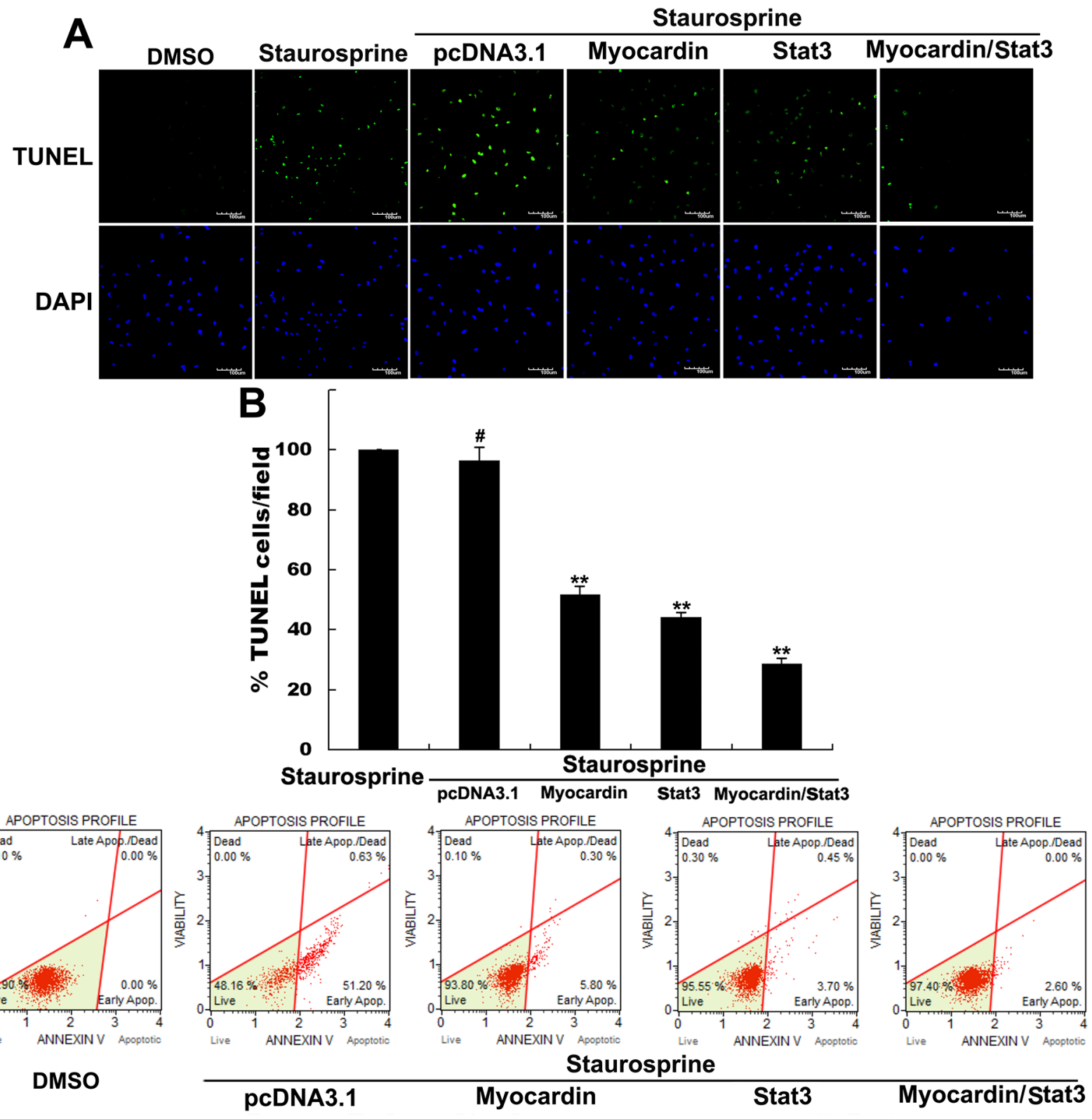

Figure 1: Myocardin and Stat3 inhibit staurosporine-induced cardiomyocyte apoptosis. (A) Afterstimulating postnatal rat cardiomyocytes with $1 \mu \mathrm{M}$ staurosporine for $30 \mathrm{~min}$ to induce cardiomyocyte apoptosis, cells were transfected with the plasmids pcDNA3.1, Myocardin, Stat3, and Myocardin/Stat3 for 24 hours. Then we used TUNEL Assay to observe the change of apoptotic rate from treatment with staurosporine or transfection with the plasmids pcDNA3.1, Myocardin, Stat3, and Myocardin/Stat3 in the presence of staurosporine. (B) Statistical software was used to analyze the change in apoptotic rate by the TUNEL Assay. ${ }^{* *}, p<0.01,{ }^{*}, p>0.05 . \mathrm{n}=3 .(\mathbf{C})$ Afterstimulating postnatal rat cardiomyocytes with $1 \mu \mathrm{M}$ staurosporine for $30 \mathrm{~min}$ induce cardiomyocyte apoptosis, cells were transfected with the plasmids pcDNA3.1, Myocardin, Stat3, and Myocardin/Stat3 for 24 hours. Annexin V-FITC apoptosis detection assay was used to observe the change of apoptotic rate by treatment with staurosporine or transfection with the plasmids pcDNA3.1, Myocardin, Stat3, and Myocardin/Stat3 in the presence of staurosporine. 


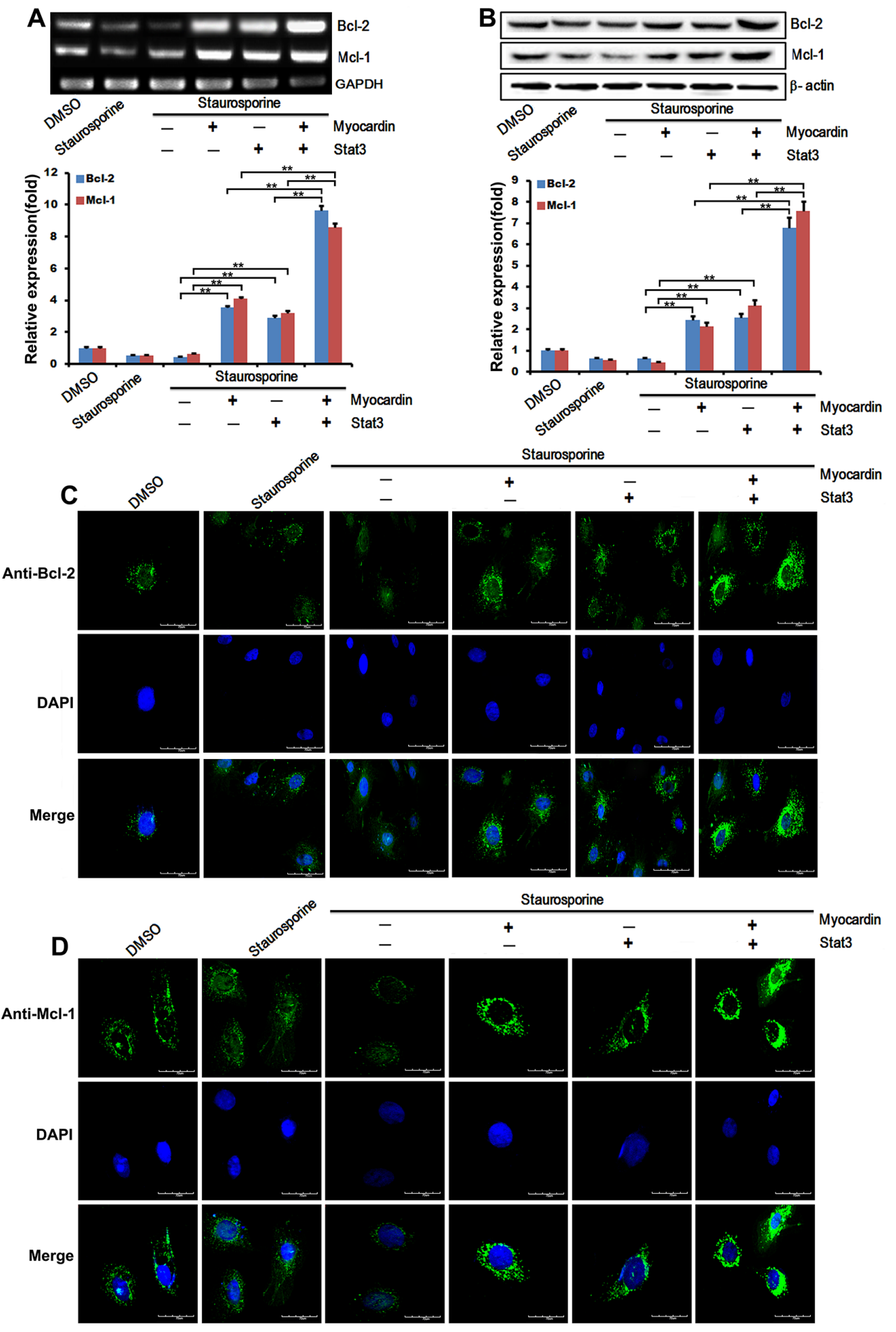

Figure 2: Myocardin and Stat3 enhance the expression of anti-apoptotic genes. (A) Afterstimulating postnatal rat cardiomyocytes with $1 \mu \mathrm{M}$ staurosporine for 30min induce cardiomyocyte apoptosis, cells were transfected with the plasmids pcDNA3.1, Myocardin, Stat3, and Myocardin/Stat3 for 24 hours. Total RNAs were isolated, and the expression of Bcl-2 and Mcl-1 was examined by RTPCR (including quantifications performed with Quantity One software). ${ }^{* *}, p<0.01$. $\mathrm{n}=3$. (B) Afterstimulating postnatal rat cardiomyocytes with $1 \mu \mathrm{M}$ staurosporine for $30 \mathrm{~min}$ induce cardiomyocyte apoptosis, cells were transfected with the plasmids pcDNA3.1, Myocardin, Stat3, and Myocardin/Stat3 for 24 hours. Total proteins were extracted, and the protein levels of Bcl-2 and Mcl-1 were detected by western blot analysis (including quantifications performed with Quantity One software). $\beta$-actin was used as a loading control. ${ }^{* *}, p<0.01 . \mathrm{n}=3$. (C and D) Representative images of immunostained cardiomyocytes. The left panels (green) respectively show anti- Bcl-2 and Mcl-1 antibody reactivity to demonstrate gross morphology. The middle panels (blue) show the DAPI staining for nuclei. The right panels respectively show double immunostaining for Bcl-2 and Mcl-1 and nuclei. Scale $=70 \mu \mathrm{m}$. 
3F). These observations indicate that both Myocardin and Stat 3 act as a potent anti-apoptotic by changing the ratio of $\mathrm{Bcl}-2 / \mathrm{Bax}$, which reduces the release of cytochrome $\mathrm{C}$ and the expression of Apaf-1, caspase- 9 , and caspase- 3 to affect the intrinsic or mitochondrial apoptotic pathway.

\section{Myocardin and Stat3 directly interact in vivo}

Myocardin, a coactivator of serum response factor (SRF) was reported to be a key regulator of cardiac and smooth muscle differentiation [17]. SRF as a direct regulator of Bcl-2 transcription as a novel mechanism is important for cell survival during embryonic development [20]. Stat3-mediated transcription of Bcl-2 and Mcl-1 prevent apoptosis in polyamine-depleted cells [35]. Since Myocardin and Stat3 can affect cells development and significantly inhibit staurosporine-induced cardiomyocyte apoptosis, we hypothesized that Myocardin and Stat3 have a direct physical interaction during cardiomyocyte apoptosis. Coimmunoprecipitation assays were used in cardiomyocytes to identify an interaction of Myocardin and Stat3 in the cellular environment. Our results
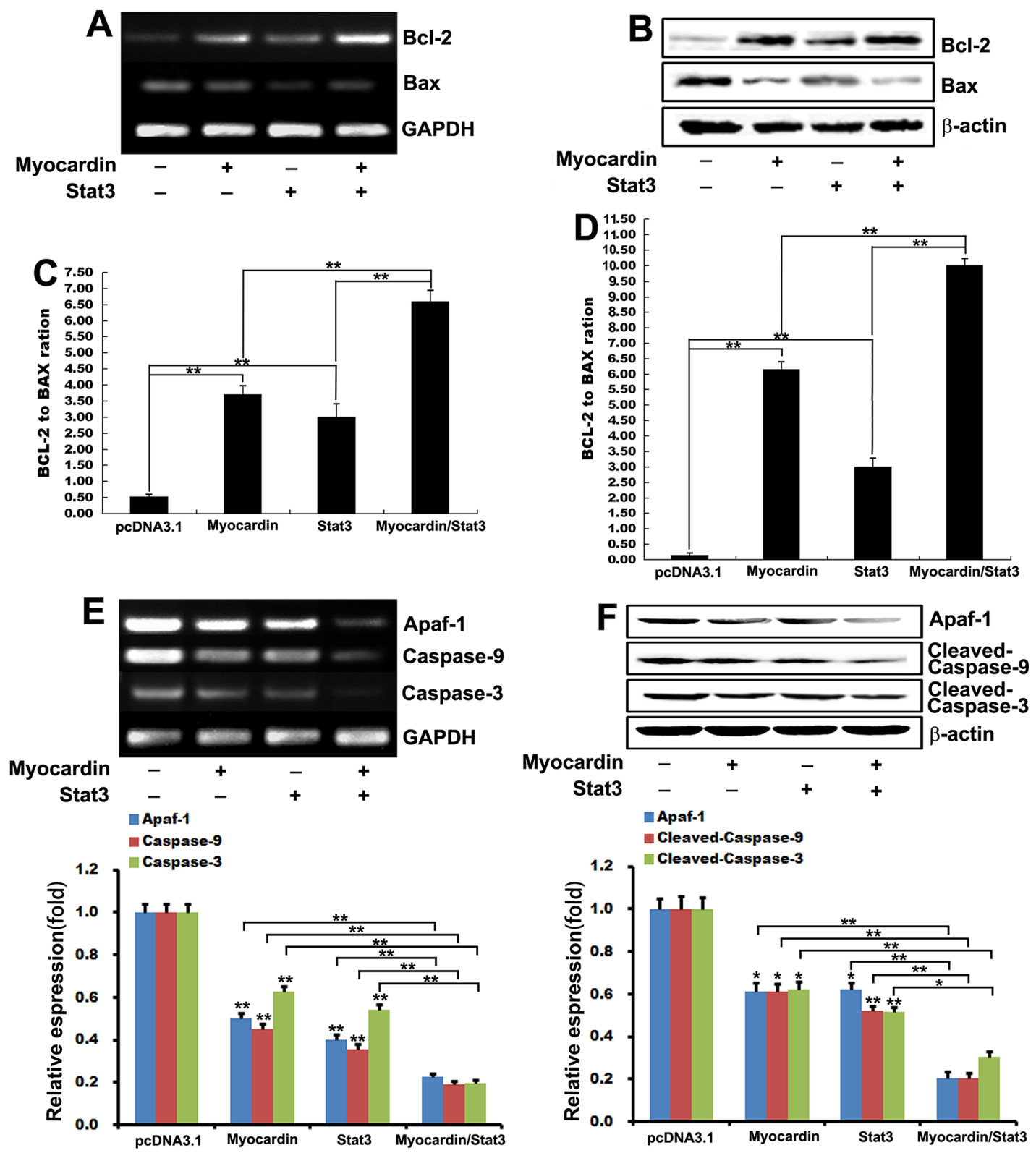

Figure 3: Myocardin and Stat3 mediated anti-apoptosis by the intrinsic or mitochondrial apoptotic pathway. (A and B) The expression of Bcl-2 and Bax was examined by RT-PCR and western blot analysis. GAPDH and $\beta$-actin were used as loading controls. (C and $\mathbf{D})$ The ratio of Bcl-2 to Bax was determined by statistical software. ${ }^{* *}, p<0.01$. $\mathrm{n}=3$. (E and F) After postnatal rat cardiomyocytes were transfected with pcDNA3.1, Myocardin, Stat3, and Myocardin/Stat3 for 24 hours, the expression of Apaf-1, caspase-9 and caspase-3 were examined by RT-PCR and western blot analysis (including quantifications performed with Quantity One software). ${ }^{* *}, p<0.01,{ }^{*}, p<0.05$. $\mathrm{n}=3$. 
demonstrate a direct interaction between Myocardin and Stat3 in vivo (Figure 4).

\section{Molecular mechanism of Myocardin and Stat3 anti-apoptosis marker gene regulation}

We constructed a luciferase promoter of Bcl-2 and Mcl-1 containing the site of CArG and measured the luciferase activity after transfection with the expression plasmid of Myocardin and Stat3 (Figure 5A and 5B) to determine whether Myocardin and Stat 3 can promote the transcription of Bcl-2 and Mcl-1. Our data show that Stat3 can lightly enhance the Bcl-2 reporter gene expression, Bcl-2 reporter gene was markedly activated by Myocardin, and cotransfection of the two plasmids significantly improved the activity of the Bcl-2 promoter (Figure 5C). Similarly, cotransfection with Myocardin and Stat3 stimulated the transcriptional activity of the Mcl-1 promoter. These data show that Myocardin and Stat3 significantly enhanced the Bcl-2 and Mcl-1 reporter activities (Figure 5D).

Myocardin drives transcription by forming a stable complex with SRF bound to the CArG box commonly found in many smooth muscle and myofibroblast gene promoters. SRF binds directly to the murine Bcl-2 regulatory region in vitro and in vivo and activates $\mathrm{Bcl}-2$ promoter-driven reporter gene transcription during early murine embryogenesis [20]. SRF binds to the cognate sites in the -107-bp region of Mcl-1 to increase the expression of Mcl-1through SRF-stimulated pathways [21]. To determine whether Myocardin/Stat3-mediated Bcl-2 and Mcl-1 promoter transactivation is dependent on the CArG box, we mutated the CArG box. As expected, CArG box mutation in the proximal promoter abolished Bcl-2 promoter activity (Figure 5C). Similarly, mutation of CArG box in the proximal promoter abolished Mcl-1 activity (Figure 5D).

ChIP assays further confirmed the impact of Myocardin/Stat3 on the Bcl-2 and Mcl-1 gene promoters. ChIP assays provided direct evidence for the involvement of Myocardin/Stat3 in the transcription of the endogenous Bcl-2 and Mcl-1 gene within the context of intact chromatin. Our results show that Myocardin and Myocardin/Stat3 bind the CArG box of the Bcl-2 promoter (Figure 5E) and the Mcl-1 promoter (Figure 5F). These results establish that the CArG box is necessary and sufficient for Myocardin mediated Bcl-2 and Mcl-1 promoter activity in cardiomyocytes.

\section{DISCUSSION}

Apoptosis is associated with loss of cardiomyocytes following myocardial infarction, atherosclerotic plaque instability, and congestive heart failure [36]. Since cardiomyocyte loss is the most important determinant of patient morbidity and mortality, fully understanding the regulatory mechanisms of apoptotic signaling is crucial. Apoptosis can be initiated by caspase-dependent or -independent mechanisms [37], and Bcl-2 family members are key regulators of the apoptotic pathway: Bcl-2, Bcl-xl, or Mcl-1 inhibit caspase activation [38]. We revealed a new role for Myocardin and Stat3-regulated apoptotic and anti-apoptotic gene expression to regulate cardiomyocyte apoptosis. We conclude that Myocardin and Stat3 have a
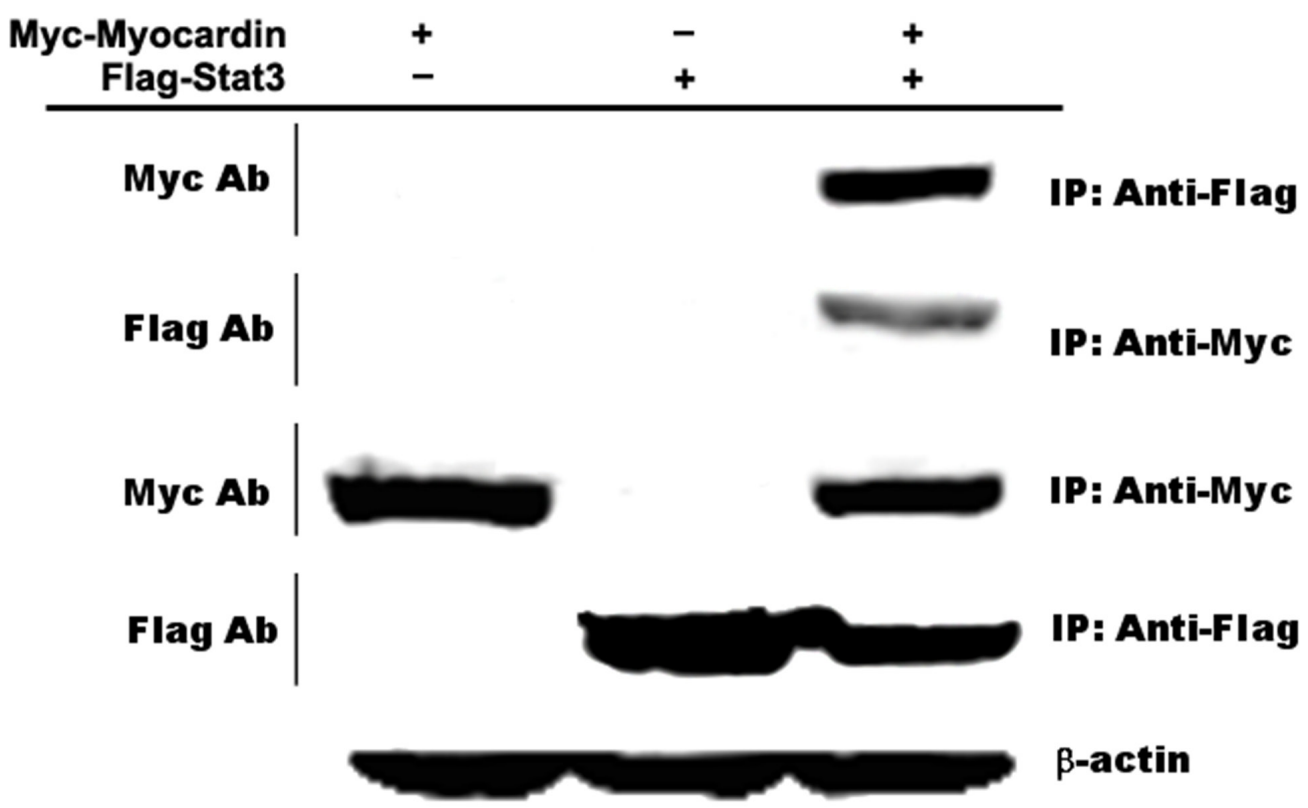

Figure 4: Myocardin and Stat3 interact in vivo. Coimmunoprecipitation assays were used to identify the interaction of Myocardin and Stat3 after cotransfection of cardiomyocytes with myc-Myocardin and flag-Stat3 for 24 hours. 
synergistic effect myocardial protection in cardiomyocyte apoptosis from our novel discovery that Myocardin and Stat 3 protect cells from apoptotic cell death by regulating anti-apoptosis gene $\mathrm{Bcl} 2$ and $\mathrm{Mcl}-1$ activity.

Stat3 signaling is an important molecular pathway that regulates cell renewal, differentiation, and apoptosis of various cell types [4-7]. Many studies have shown that Stat 3 plays oncogenic roles by promoting the expression of cancer-associated genes such as cyclinD1, c-Myc, Cox2, and Bcl-2 [39-42]. Stat3-mediated transcription of Bcl$2, \mathrm{Mcl}-1$, and c-IAP2 prevents tumor necrosis factor- $\alpha$ (TNF- $\alpha$ )-induced apoptosis in polyamine-depleted cells [35]. However, TNF- $\alpha$ induced cardiomyocyte apoptosis is mitigated by IL-10 treatment via the upregulation of Akt phosphorylation that further increases Stat3 phosphorylation [43]. Constitutive cardiomyocyterestricted deletion of Stat3 has been found to result in increased apoptosis and increased susceptibility to doxorubicin-induced heart failure [13]. Our data showed that Stat3 significantly weakened the cardiomyocyte apoptosis rate induced by staurosporine and promoted the expression of the anti-apoptotic genes Bcl-2 and Mcl-1.

Myocardin is expressed specifically in cardiac and smooth muscle cells and potently activates their gene expression by associating with SRF bound to CArG boxes [17-19]. Previous studies have demonstrated that SRF promotes differentiating murine embryonic stem (ES) cell survival in vitro by binding to the Bcl-2 promoter in vivo and activating Bcl-2 transcription [20]. Our present study demonstrated that Myocardin also promotes cell survival by regulating the expression of anti-apoptotic genes. Firstly, we found that Myocardin inhibits staurosporine-induced cardiomyocyte apoptosis by TUNEL assay and Annexin V-FITC apoptosis detection assay. Cardiomyocyte cells transfected with Myocardin had reduced annexin- $\mathrm{V}$ expression on the cell surface as compared to control, which implicates Myocardin in cardiomyocyte apoptosis (Figure 1). The Bcl-2 family, consisting of both pro-apoptotic and anti-apoptotic proteins, is a critical checkpoint of the apoptosis pathway
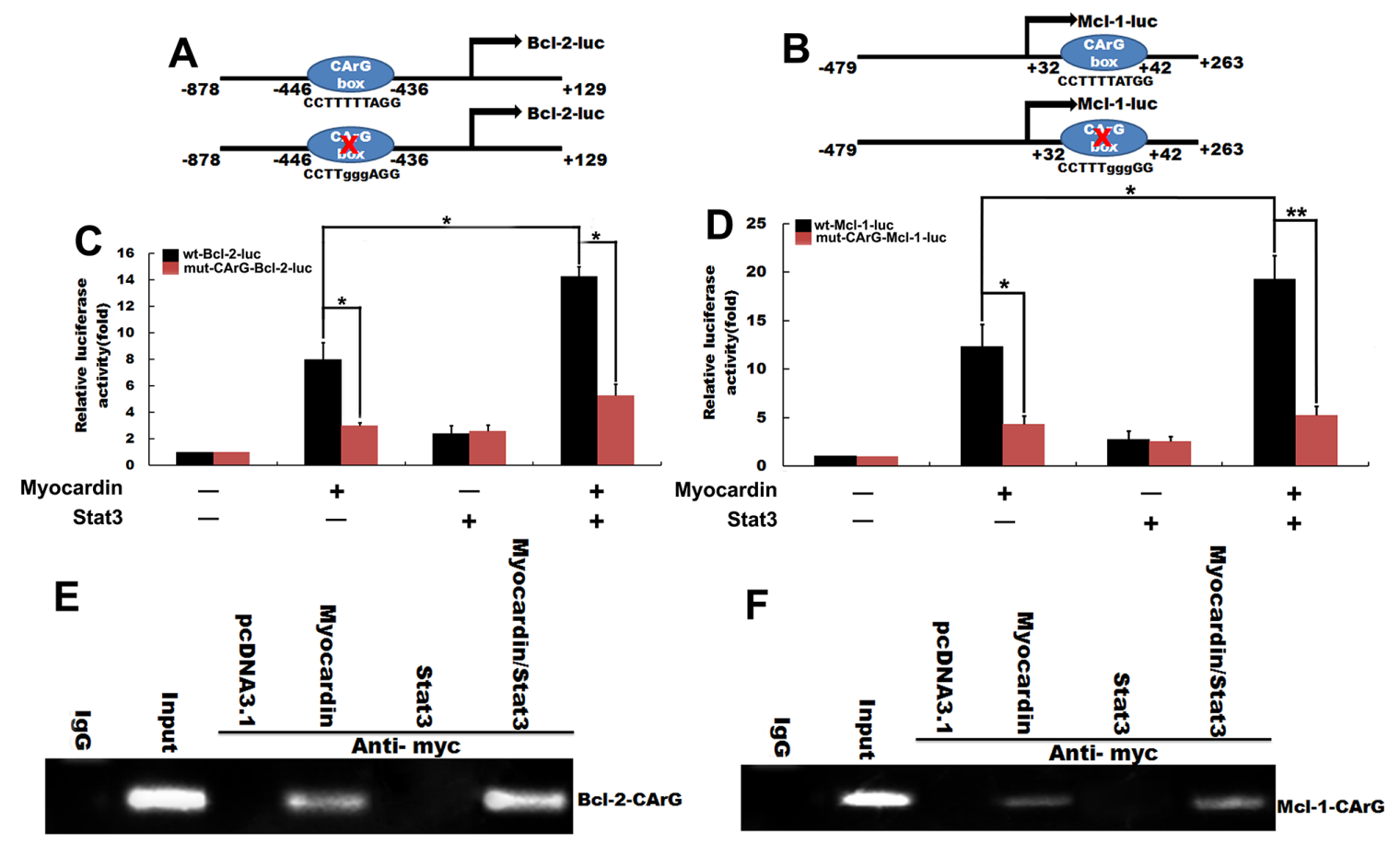

Figure 5: The molecular mechanism of Myocardin and Stat3 ranti-apoptosis marker gene regulation. (A) Schematic of the $-878 \mathrm{Bcl}-2$ promoter, containing the CArG box element, which was linked to a luciferase reporter and the $-878 \mathrm{Bcl}-2$ promoter with a mutation in CArG box. (B) The postnatal rat cardiomyocytes were transfected with the wild-type $-878 \mathrm{Bcl}-2$ promoter, or the $-878 \mathrm{Bcl}-2$ promoter with a mutation in CArG box, and transfected with pcDNA3.1, Myocardin, Stat3, and Myocardin/Stat3 for 24 hours. Luciferase reporter assay was used to test the transactivity of Bcl-2. (C) Schematic of the $-479 \mathrm{Mcl}-1$ promoter, containing CArG box elements and the -479 Mcl-1promoter with mutation CArG box. ${ }^{*}, p<0.05$. $\mathrm{n}=6$. (D) Postnatal rat cardiomyocytes were transfected with the wild-type $-479 \mathrm{Mcl}-1$ promoter, or the $-479 \mathrm{Mcl}-1$ promoter with a mutation in CArG box, and transfected with pcDNA3.1, Myocardin, Stat3, and Myocardin/Stat3 for 24 hours. Then the luciferase reporter assay was used to test the transactivity of Mcl-1. ${ }^{* *}, p<0.01,{ }^{*}, p<0.05 . \mathrm{n}=6 .(\mathbf{E}$ and F) The postnatal rat cardiomyocytes were transiently transfected with pcDNA3.1, Myocardin, Stat3, and Myocardin/Stat3 for 24 hours. ChIP assays were performed with primers for sequences associated with the genes for Bcl-2 and Mcl-1. Sheared DNA/protein complexes were immunoprecipitated by using an anti-myc Ab. Then, PCR was used to detect the endogenous CArG regions in immunoprecipitated chromatin fragments. The amount of DNA in each sample (input) is shown at the second land. Immunoprecipitations were performed with IgG as a negative control. 
[44]. Mcl-1 has also been found to exhibit both similarities to and differences from Bcl-2 [45]. We further investigated the effect of Myocardin in regulating the expression of Bcl-2 and Mcl-1 in the presence of staurosporine. Our data showed that the expression of both Bcl-2 and Mcl1 increased slightly after transfection with Myocardin. Based on these findings we concluded that Myocardin could weaken staurosporine-induced cardiomyocyte apoptosis and increase expression of anti-apoptotic Bcl2 and Mcl-1. Importantly, cotransfected Myocardin and Stat 3 to cardiomyocytes obviously reduced the number of apoptotic cells. Moreover, cotransfection of Myocardin and Stat3 synergistically increased the mRNA levels and protein levels of anti-apoptotic proteins Bcl-2 and Mcl-1 (Figure 2A and 2B). These observations support Myocardin and Stat 3 mediated cardiomyocyte protection from apoptosis through enhancement of anti-apoptotic gene expression.

To clarify the regulatory mechanism we also identified the direct physical interaction of Myocardin and Stat 3 by coimmunoprecipitation assays in cardiomyocytes. We found that transcription factors Myocardin and Stat3 interact directly in vivo (Figure 4). Given that the ratio of anti-apoptotic proteins such as Bcl-2 relative to procell death proteins such as Bax determines the ultimate sensitivity of cells to various apoptotic stimuli [46], we calculated the ratio of Bcl-2 expression to Bax expression in the present study. The densitometric analysis revealed a high Bcl-2/Bax ratio in transfected Myocardin and Stat 3 cardiomyocytes. From these findings, we conclude that Myocardin and Stat 3 increase the expression of Bcl2 while reducing the pro-apoptotic gene-Bax, which changes the ratio of $\mathrm{Bcl}-2 / \mathrm{Bax}$ to regulate the process of cardiomyocyte apoptosis.

Next, we constructed a luciferase promoter of Bcl-2 and Mcl-1 containing the site of CArG to study the mechanism of Myocardin and Stat3 antiapoptosis marker gene regulation. Our data show that the cotransfection of Myocardin and Stat3 significantly enhance the transcriptional activity of Bcl-2 (Figure 2). Similarly, Myocardin and Stat3 significantly promote the transcriptional activity of Mcl-1. It has been demonstrated that SRF binds to the cognate sites in the -107-bp region of the Mcl-1 to increase the expression of Mcl-1through SRF-stimulated pathways [21]. The CArG box in the $\mathrm{Bcl}-2$ promoter motif is necessary for activation of Bcl-2 transcription by SRF in ES cells in vivo [20]. We determined whether Myocardin/Stat3-mediated Bcl-2 and $\mathrm{Mcl}-1$ promoter transcriptional activation depend on the CArG box. We found that mutation of CArG box in the proximal promoter abolished Myocardin/Stat3 stimulated Bcl-2 and Mcl-1 promoter activity in vitro. This observation confirmed our hypothesis that Myocardin/ Stat3 promotion transactivity of Bcl-2 and Mcl-1 is dependent on CArG box. ChIP assays provide consistent and direct evidence of Myocardin/Stat3 involvement in endogenous Bcl-2 and Mcl-1 gene transcription within the context of the intact $\mathrm{CArG}$ box (Figure 5E and 5F).

In summary, the present study reveals a novel Myocardin/Stat3 anti-apoptotic gene signaling pathway that is operative during cardiomyocyte apoptosis and a molecular explanation for Myocardin/Stat3 inhibition of cardiomyocyte apoptosis and myocardial protection. Cardiomyocyte apoptosis is a crucial process in heart disease, including the progression from the initiation of hypertrophy (compensatory phase) to decompensation and transition to heart failure. Mechanistic insight into cardiomyocyte apoptosis regulation has led to novel therapeutic approaches. Using Myocardin/Stat3 (and analogs) to inhibit cardiomyocyte apoptosis holds promise as an effective therapeutic strategy for cardiovascular diseases.

\section{MATERIALS AND METHODS}

\section{Plasmids, cell culture, cell treated, cell transfection}

Myc-pcDNA3.1-Myocardin encoded the full-length mouse Myocardin, and flag-pcDNA3.1-Stat3 encoded the full-length mouse Stat3. Bcl-2 (-878 to +129) or Mcl-1 $(-479$ to +263$)$ luciferase reporters were constructed in pGL3-basic plasmid (Promega).

The primers used to create Bcl-2-luc and Mcl-1luc were as follows: Bcl-2: 5' GGGTGGTACCAGGAG GGCTCTTTCTTTCTTCTTTT 3' (sense) and 5' GGCA AGCTTCCCATCAATCTTCAGCACTCTCCAG3' (antisense); Mcl-1: 5' CT CTCGAGAATGGTTCTTT AGGGTAGCACGTGG3' (sense) and 5' CAAAGCTT CCCCCCCACAGTAGAGGTTGAGTC3' (antisense);

Mutations of the CArG sequences were performed with the QuickChange Site-directed Mutagenesis Kit (Stratagene) using the following mutagenesis primers:

Bcl-2 CArG mut: F: 5'-AACTTCGTAGCAGTCA TCCTTgggAGGAAAAGAGGGAAAAAATAA-3' Bcl-2 CArG mut: R: 5'-TTATTTTTTCCCTCTTT TCCTCCCAAGGATGACTGCTACGAAGTT-3'; Mcl-1 CArG mut: F: 5'-AAGCTGCCGCCCCTT TCCCCTTTggg GGGAATACTTTTTTGGCGGC| GCGCG-3';

Mcl-1 CArG mut: R: 5'-CGCGCGCCGCCAAAAA AGTATTCCCCCCAAAGGGGAAAGGGGCGGC AGCTT-3';

Cardiomyocytes were isolated from d1-3 SpragueDawley rat pups as described previously [47-50], starved without serum for another 12 hours, and plated in growth medium without antibiotics. Cells were treated at 30$50 \%$ confluency with $1 \mu \mathrm{M}$ staurosporine (Sigma) for 30 $\mathrm{min}$ to induce cardiomyocyte apoptosis. Cardiomyocytes were transfected with plasmids pcDNA3.1, Myocardin, Stat3, and Myocardin/Stat3 with FuGENE ${ }^{\circledR} H D$ (Roche) according to manufacturer instructions. After transfection, 
the medium was removed and replaced with normal culture medium.

\section{RNA isolation and semiquantitative reverse- transcription polymerase chain reaction (RT- PCR)}

Semiquantitative RT-PCR analysis was carried out as described previously [50]. Briefly, total RNA was isolated from cells using Trizol reagent (Invitrogen), the samples were reverse-transcribed by using M-MLV reverse transcriptase (Invitrogen) according to the manufacturer's instructions. The PCR primer sequences are as follows:

GAPDH: F-ATTCAACGGCACAGTCAAGG, R-GCAGAAGGGGCGGAGATGA; Bcl-2: F-CGGGAGAACAGGGTATGA, R-CAGG CTGGAAGGAGAAGAT; Mcl-1: F-AAGAGGCTGGGATGGGTT，R-TTG GTGGCTGGAGGTTTT;

Bax: F-TGGTTGCCCTCTTCTAC, R-AGCCA CCCTGGTCTTG;

Cytochrome c: F-GGCAAGCATAAGACTGGA, R-GGTCTGCCCTTTCTCCCT; Apaf-1: F-CCTGAAGCCCAAGTGAGT, R-TGC CCTGATGACAAGTAAAG; Caspase-9: F-CCCCACCCTCACTTTGCT, R-TAA GTGAGCCTGGTCCTCC; Caspase-3: F-CTGGACTGCGGTATTGAG, R-GG GTGCGGTAGAGTAAGC;

Semiquantitative analysis of mRNA expression was performed with Biorad software. Glyceraldehyde-3phosphate dehydrogenase (GAPDH) expression was used as an internal control.

\section{Protein isolation and western blotting}

The procedure for Western Blotting has been described previously [51]. After treatment, cells were lysed in lysis buffer. Proteins were separated by SDS-PAGE, then transferred to an $\mathrm{NC}$ membrane (Pall) and blocked for $60 \mathrm{~min}$ at room temperature in 5\% skim milk powder (wt/ vol) and PBST (PBS + 0.05\% Tween-20, vol/vol). After incubation with mouse polyclonal antibodies (goat antimouse Bcl-2 (Abcam), goat anti-mouse Bax (Abcam), goat anti- mouse Mcl-1 (Abcam), goat anti- rabbit Apaf-1 (Cell Signaling Technology), goat anti- rabbit Cleavedcaspase-9 (Cell Signaling Technology), and goat antirabbit Cleaved-caspase-3 (Cell Signaling Technology), the appropriate secondary antibodies (IRDye ${ }^{\mathrm{TM}} 800$-goat antimouse, IRDye ${ }^{\mathrm{TM}} 800$-goat anti-rabbit, IRDye ${ }^{\mathrm{TM}} 680$-goat anti-mouse, IRDye ${ }^{\mathrm{TM}}$ 680- goat anti-rabbit, Li-COR) were added for incubation. Specific proteins were visualized with an Odyssey Infrared Imaging System. Mouse anti $\beta$-actin (Santa Cruz) expression was used as an internal control.

\section{Apoptosis assay}

Apoptosis was determined through externalization of plasma membrane phosphatidylserine (PS) and TUNEL assay analysis

\section{Annexin V-FITC apoptosis detection assay}

An increase in the plasma membrane PS externalization occurs early in apoptosis and can be detected by Annexin V staining. Cardiomyocytes were treated with staurosporine $30 \mathrm{~min}$ as described above, then transfected with plasmids pcDNA3.1, Myocardin, Stat3, and Myocardin/Stat3 for 24 hours. Cardiomyocytes were isolated, stained with annexin V-FITC (BioVision Research Products, Mountain View, CA), and analyzed using FACS (BD Accuri C6, USA) for fluorescence of annexin V-positive cells.

\section{TUNEL assay for cardiomyocyte apoptosis}

The terminal deoxynucleotidyl transferase-mediated dUTP nick-end labeling (TUNEL) assay was used to monitor the extent of DNA fragmentation as a measure of apoptosis in paraffin-embedded sections. Cardiomyocytes were fixed with formaldehyde. Immunohistochemical detection of apoptotic cells was carried out with a TUNEL reaction by using DeadEnd ${ }^{\mathrm{TM}}$ Fluorometric TUNEL System (Promega). The cells were washed 3 times with PBS, blocked with $10 \%$ normal goat serum in PBS, and incubated with DAPI (4',6'-diamidino-2-phenylindole) to stain the nuclei. The samples were evaluated under inverted confocal laser microscope (Olympus, Japan). For the quantitative purpose, TUNEL-positive cardiomyocytes were counted via microscope.

\section{Coimmunoprecipitation}

Plasmid-based expression vectors encoding myc-tagged myocardin and flag-tagged Stat3 were cotransfected into cardiomyocytes. Lysates were collected 48 hours post-transfection. Then myc antibody (Santa Cruz) was used to precipitate myc-myocardin, and flag antibody (Santa Cruz) was used to precipitate flagStat3. The resulting mixture was washed, separated by SDS-PAGE, and transferred to an NC membrane (Pall). Samples were probed with myc antibody to visualize flagepitope Stat3, stripped, and probed with flag antibody to visualize myc-epitope Myocardin.

\section{ChIP assay}

ChIP analysis was performed using a commercially available kit (Enzymatic Chromatin IP (Magnetic Beads), Cell Signaling Technology, USA) on cardiomyocytes transfected with Myocardin, Stat3, and Myocardin/Stat3 for 24 hours. DNA-bound proteins 
were cross-linked using formaldehyde at a final concentration of $1 \%$ for $20 \mathrm{~min}$ at room temperature. Protein-DNA complexes were immunoprecipitated using primary antibodies for myc antibody (Santa Cruz). Myocardin and Mcl-1 promoter complexes, Stat3 and Mcl-1 promoter complexes, and Myocardin, Stat3 and Mcl-1 promoter complexes were measured by PCR. The primers used for the amplification of the human promoter region between -550 to $-311 \mathrm{bp}$ were: $\mathrm{Bcl}-2$ CArG box forward 5'GCAGGACCAGGAGGAGGA 3', reverse 5' GATAAATGAAGGCAGGACGC 3'; human Mcl-1 promoter region between -113 to +140bp were: Mcl-1-CArG box forward 5, CACTCAGAGCCTCCGAAGACC 3', and reverse 5' GAAGTGAGAAGTGGCGAGCAG 3'. The samples were separated by electrophoresis in $2 \%$ agarose gel and visualized by ethidium bromide staining.

\section{Immunocytochemistry assay}

Immunofluorescence assays were performed as described previously [52]. After adding the primary antibodies (mouse anti-Bcl-2(Abcam), mouse anti-Mcl$1($ Abcam $)$ ), we added appropriate secondary antibodies (FITC-goat anti-mouse IgG Santa Cruz) to the samples. DAPI was used to stain nuclei. The samples were then evaluated under an inverted laser scanning confocal microscope (OLYMPUS-FV-1000, Japan).

\section{Luciferase assay}

Luciferase assays were performed as described previously [51]. Cardiomyocytes were seeded onto 24-well plates (Corning). Transfections were performed with FuGENE ${ }^{\circledR} \mathrm{HD}$ (Roche) according to manufacturer protocol. The total amount of DNA per well was kept constant by adding the corresponding amount of expression vector without a cDNA insert. After transfection for 24 hours, luciferase activity was measured with a luciferase reporter assay system (Promega) on a Synergy ${ }^{\mathrm{TM}} 4$ Hybrid Microplate Reader (Biotek). Transfection efficiencies were normalized to the total protein concentration of each luciferase assay preparation. All experiments were performed in triplicate with different preparations of plasmids and primary cells. Data of representative experiments are presented as the mean \pm standard deviation of triplicates.

\section{Statistical analysis}

Data are presented as mean $\pm \mathrm{SE}$ and accompanied by the number of experiments performed independently, and analyzed by $t$-test. Differences with $P<0.05$ were considered statistically significant.

\section{Abbreviations}

Stat3, Signal transducer and activator of transcription 3; Mcl-1, myeloid cell leukemia-1; Apaf-1, apoptotic protease activating factor-1; TNF- $\alpha$, tumor necrosis factor- $\alpha$; ES, embryonic stem; GAPDH, Glyceraldehyde3-phosphate dehydrogenase; PS, phosphatidylserine; TUNEL, The terminal deoxynucleotidyl transferasemediated dUTP nick-end labeling; DAPI, 4',6'-diamidino2-phenylindole.

\section{Author contributions}

X.H.L. and T.C.Z. designed research; X.Y. and X.H.L. performed research; J.P.L., H.L., H.Q., A.Y., C.X.Y., and W.G. analyzed data; and X.Y., X.H.L., H.P., C.J.G., and T.C.Z. wrote the paper.

\section{ACKNOWLEDGMENTS}

This work was financially supported by National Natural Science Foundation of China (No. 31501149, 31570764, 31401117, 31471282, 31440038, 31270837). Hubei Province health and family planning scientific research project (WJ2017M173) and the Science and Technology Young Training Program of the Wuhan University of Science and Technology (2016xz035 and 2017xz027) and the innovation and entrepreneurship fund for Graduate of Wuhan University of Science and Technology.

\section{CONFLICTS OF INTEREST}

No conflicts of interest.

\section{REFERENCES}

1. Kang PM, Izumo S. Apoptosis in heart: basic mechanisms and implications in cardiovascular diseases. Trends in molecular medicine. 2003; 9:177-182.

2. Kim NH, Kang PM. Apoptosis in cardiovascular diseases: mechanism and clinical implications. Korean circulation journal. 2010; 40:299-305.

3. Colucci WS. Apoptosis in the heart. The New England journal of medicine. 1996; 335:1224-1226.

4. Bromberg J, Darnell JE. The role of STATs in transcriptional control and their impact on cellular function. Oncogene. 2000; 19:2468.

5. Yu H, Jove R. The STATs of cancer-new molecular targets come of age. Nature Reviews Cancer. 2004; 4:97-105.

6. Yasukawa H, Hoshijima M, Gu Y, Nakamura T, Pradervand S, Hanada T, Hanakawa Y, Yoshimura A, Ross J, Chien KR. Suppressor of cytokine signaling-3 is a biomechanical stress-inducible gene that suppresses gp130-mediated cardiac myocyte hypertrophy and survival pathways. The Journal of clinical investigation. 2001; 108:1459-1467. 
7. Funamoto M, Fujio Y, Kunisada K, Negoro S, Tone E, Osugi T, Hirota H, Izumi M, Yoshizaki K, Walsh K. Signal transducer and activator of transcription 3 is required for glycoprotein 130-mediated induction of vascular endothelial growth factor in cardiac myocytes. Journal of Biological Chemistry. 2000; 275:10561-10566.

8. Zushi S, Shinomura Y, Kiyohara T, Miyazaki Y, Kondo S, Sugimachi M, Higashimoto Y, Kanayama S, Matsuzawa Y. STAT 3 mediates the survival signal in oncogenicrastransfected intestinal epithelial cells. International journal of cancer. 1998; 78:326-330.

9. Bromberg JF, Wrzeszczynska MH, Devgan G, Zhao Y, Pestell RG, Albanese C, Darnell JE. Stat3 as an oncogene. Cell. 1999; 98:295-303.

10. Rahaman SO, Harbor PC, Chernova O, Barnett GH, Vogelbaum MA, Haque SJ. Inhibition of constitutively active Stat 3 suppresses proliferation and induces apoptosis in glioblastoma multiforme cells. Oncogene. 2002; 21:8404.

11. Aoki Y, Feldman GM, Tosato G. Inhibition of STAT3 signaling induces apoptosis and decreases survivin expression in primary effusion lymphoma. Blood. 2003; 101:1535-1542.

12. Hilfiker-Kleiner D, Hilfiker A, Fuchs M, Kaminski K, Schaefer A, Schieffer B, Hillmer A, Schmiedl A, Ding Z, Podewski E. Signal transducer and activator of transcription 3 is required for myocardial capillary growth, control of interstitial matrix deposition, and heart protection from ischemic injury. Circulation research. 2004; 95:187-195.

13. Jacoby JJ, Kalinowski A, Liu MG, Zhang SM, Gao Q, Chai GX, Ji L, Iwamoto Y, Li E, Schneider M. Cardiomyocyterestricted knockout of STAT3 results in higher sensitivity to inflammation, cardiac fibrosis, and heart failure with advanced age. Proceedings of the National Academy of Sciences. 2003; 100:12929-12934.

14. Wang Z, Wang DZ, Pipes GC, Olson EN. Myocardin is a master regulator of smooth muscle gene expression. Proceedings of the National Academy of Sciences of the United States of America. 2003; 100:7129-7134.

15. Small EM, Warkman AS, Wang DZ, Sutherland LB, Olson EN, Krieg PA. Myocardin is sufficient and necessary for cardiac gene expression in Xenopus. Development. 2005; 132:987-997.

16. Cao D, Wang C, Tang R, Chen H, Zhang Z, Tatsuguchi $\mathrm{M}$, Wang DZ. Acetylation of myocardin is required for the activation of cardiac and smooth muscle genes. Journal of Biological Chemistry. 2012; 287:38495-38504.

17. Pipes GT, Creemers EE, Olson EN. The myocardin family of transcriptional coactivators: versatile regulators of cell growth, migration, and myogenesis. Genes \& development. 2006; 20:1545-1556.

18. Yoshida T, Sinha S, Dandré F, Wamhoff BR, Hoofnagle MH, Kremer BE, Wang DZ, Olson EN, Owens GK. Myocardin is a key regulator of CArG-dependent transcription of multiple smooth muscle marker genes. Circulation research. 2003; 92:856-864.

19. Sun Q, Taurin S, Sethakorn N, Long X, Imamura M, Wang DZ, Zimmer WE, Dulin NO, Miano JM. Myocardindependent activation of the CArG box-rich smooth muscle gamma-actin gene: preferential utilization of a single CArG element through functional association with the NKX3.1 homeodomain protein. The Journal of biological chemistry. 2009; 284:32582-32590.

20. Schratt G, Philippar U, Hockemeyer D, Schwarz H, Alberti S, Nordheim A. SRF regulates Bcl-2 expression and promotes cell survival during murine embryonic development. The EMBO journal. 2004; 23:1834-1844.

21. Townsend KJ, Zhou P, Qian L, Bieszczad CK, Lowrey CH, Yen A, Craig RW. Regulation of MCL1 through a serum response factor/Elk-1-mediated mechanism links expression of a viability-promoting member of the BCL2 family to the induction of hematopoietic cell differentiation. Journal of Biological Chemistry. 1999; 274:1801-1813.

22. Tang RH, Zheng XL, Callis TE, Stansfield WE, He J, Baldwin AS, Wang DZ, Selzman CH. Myocardin inhibits cellular proliferation by inhibiting NF- $\mathrm{B}$ (p65)-dependent cell cycle progression. Proceedings of the National Academy of Sciences. 2008; 105:3362-3367.

23. Liu N, Olson EN. Coactivator control of cardiovascular growth and remodeling. Current opinion in cell biology. 2006; 18:715-722.

24. Torrado M, Iglesias R, Centeno A, López E, Mikhailov AT. Targeted gene-silencing reveals the functional significance of myocardin signaling in the failing heart. PLoS One. 2011; 6:e26392.

25. Huang J, Lu MM, Cheng L, Yuan LJ, Zhu X, Stout AL, Chen M, Li J, Parmacek MS. Myocardin is required for cardiomyocyte survival and maintenance of heart function. Proceedings of the National Academy of Sciences. 2009; 106:18734-18739.

26. Husari AW, Dbaibo GS, Bitar H, Khayat A, Panjarian S, Nasser M, Bitar FF, El-Sabban M, Zaatari G, Mroueh SM. Apoptosis and the activity of ceramide, Bax and Bcl-2 in the lungs of neonatal rats exposed to limited and prolonged hyperoxia. Respiratory research. 2006; 7:100.

27. Sawada M, Nakashima S, Banno Y, Yamakawa H, Hayashi K, Takenaka K, Nishimura Y, Sakai N, Nozawa Y. Ordering of ceramide formation, caspase activation, and $\mathrm{Bax} / \mathrm{Bcl}-2$ expression during etoposide-induced apoptosis in C6 glioma cells. Cell death and differentiation. 2000; 7:761.

28. Krajewski S, Krajewska M, Shabaik A, Miyashita T, Wang $\mathrm{HG}$, Reed JC. Immunohistochemical determination of in vivo distribution of Bax, a dominant inhibitor of Bcl-2. The American journal of pathology. 1994; 145:1323.

29. Yamamoto K, Tomita N, Yoshimura S, Nakagami H, Taniyama Y, Yamasaki K, Ogihara T, Morishita R. Hypoxiainduced renal epithelial cell death through caspasedependent pathway: role of Bcl-2, Bcl-xL and Bax in 
tubular injury. International journal of molecular medicine. 2004; 14:633-640.

30. Filippovich IV, Sorokina NI, Lisbona A, Chérel M, Chatal JF. Radiation-induced apoptosis in human myeloma cell line increases BCL-2/BAX dimer formation and does not result in BAX/BAX homodimerization. International journal of cancer. 2001; 92:651-660.

31. Ahmed MM, Alcock RA, Chendil D, Dey S, Das A, Venkatasubbarao K, Mohiuddin M, Sun L, Strodel WE, Freeman JW. Restoration of transforming growth factor- $\beta$ signaling enhances radiosensitivity by altering the Bcl-2/ Bax ratio in the p53 mutant pancreatic cancer cell line MIA PaCa-2. Journal of Biological Chemistry. 2002; 277:2234-2246.

32. Adachi S, Gottlieb RA, Babior BM. Lack of release of cytochrome $\mathrm{C}$ from mitochondria into cytosol early in the course of Fas-mediated apoptosis of Jurkat cells. Journal of Biological Chemistry. 1998; 273:19892-19894.

33. Pérez-Pinzón MA, Xu GP, Born J, Lorenzo J, Busto R, Rosenthal M, Sick TJ. Cytochrome C is released from mitochondria into the cytosol after cerebral anoxia or ischemia. Journal of Cerebral Blood Flow \& Metabolism. 1999; 19:39-43.

34. Kuida K, Haydar TF, Kuan CY, Gu Y, Taya C, Karasuyama H, Su MS, Rakic P, Flavell RA. Reduced apoptosis and cytochrome c-mediated caspase activation in mice lacking caspase 9. Cell. 1998; 94:325-337.

35. Bhattacharya S, Ray RM, Johnson LR. STAT3-mediated transcription of Bcl-2, Mcl-1 and c-IAP2 prevents apoptosis in polyamine-depleted cells. Biochemical Journal. 2005; 392:335-344.

36. Wolters S, Corsten M, Reutelingsperger C, Narula J, Hofstra L. Cardiovascular molecular imaging of apoptosis. European journal of nuclear medicine and molecular imaging. 2007; 34:86-98.

37. Prunet C, Lemaire-Ewing S, Ménétrier F, Néel D, Lizard G. Activation of caspase-3-dependent andindependent pathways during 7-ketocholesterol-and 7 $\beta$-hydroxycholesterol-induced cell death: A morphological and biochemical study. Journal of biochemical and molecular toxicology. 2005; 19:311-326.

38. Tzifi F, Economopoulou C, Gourgiotis D, Ardavanis A, Papageorgiou S, Scorilas A. The role of BCL2 family of apoptosis regulator proteins in acute and chronic leukemias. Advances in hematology. 2011; 2012.

39. Li H, Xiao W, Ma J, Zhang Y, Li R, Ye J, Wang X, Zhong $\mathrm{X}$, Wang S. Dual high expression of STAT3 and cyclinD1 is associated with poor prognosis after curative resection of esophageal squamous cell carcinoma. International journal of clinical and experimental pathology. 2014; 7:7989.

40. Voss T, Barth SW, Rummel C, Gerstberger R, Hübschle T, Roth J. STAT3 and COX-2 activation in the guinea-pig brain during fever induced by the Toll-like receptor-3 agonist polyinosinic: polycytidylic acid. Cell and tissue research. 2007; 328:549.

41. Barré B, Avril S, Coqueret O. Opposite regulation of Myc and $\mathrm{p} 21$ waf1 transcription by STAT3 proteins. The Journal of biological chemistry. 2014; 289:16643.

42. Yu LJ, Wu ML, Li H, Chen XY, Wang Q, Sun Y, Kong QY, Liu J. Inhibition of STAT3 expression and signaling in resveratrol-differentiated medulloblastoma cells. Neoplasia. 2008; 10:736-744.

43. Dhingra S, Bagchi AK, Ludke AL, Sharma AK, Singal PK. Akt regulates IL-10 mediated suppression of TNF $\alpha$ induced cardiomyocyte apoptosis by upregulating Stat3 phosphorylation. PLoS One. 2011; 6: e25009.

44. Spets H, Strömberg T, Georgii-Hemming P, Siljason J, Nilsson K, Jernberg-Wiklund H. Expression of the bcl-2 family of pro-and anti-apoptotic genes in multiple myeloma and normal plasma cells. European journal of haematology. 2002; 69:76-89.

45. Ertel F, Nguyen M, Roulston A, Shore GC. Programming cancer cells for high expression levels of Mcl1. EMBO reports. 2013; 14:328-336.

46. Zha H, Aimé-Sempé C, Sato T, Reed JC. Proapoptotic protein Bax heterodimerizes with $\mathrm{Bcl}-2$ and homodimerizes with Bax via a novel domain (BH3) distinct from BH1 and BH2. Journal of Biological Chemistry. 1996; 271:7440-7444.

47. Mitcheson JS, Hancox JC, Levi AJ. Cultured adult cardiac myocytes. Cardiovascular research. 1998; 39:280-300.

48. Suzuki T, Tsuruda A, Katoh S, Kubodera A, Mitsui Y. Purification of endothelin from a conditioned medium of cardiac fibroblastic cells using beating rate assay of myocytes cultured in a serum-free medium. Journal of molecular and cellular cardiology. 1997; 29:2087-2093.

49. Lim HW, New L, Han J, Molkentin JD. Calcineurin enhances MAPK phosphatase-1 expression and p38 MAPK inactivation in cardiac myocytes. Journal of Biological Chemistry. 2001; 276:15913-15919.

50. Liao XH, Wang N, Liu QX, Qin T, Cao B, Cao DS, Zhang TC. Myocardin-related transcription factor-A induces cardiomyocyte hypertrophy. IUBMB life. 2011; 63:54-61.

51. Liao XH, Lu DL, Wang N, Liu LY, Wang Y, Li YQ, Yan TB, Sun XG, Hu P, Zhang TC. Estrogen receptor $\alpha$ mediates proliferation of breast cancer $\mathrm{MCF}-7$ cells via a $\mathrm{p} 21 /$ PCNA/E2F1-dependent pathway. The FEBS journal. 2014; 281:927-942.

52. Liao XH, Wang N, Zhao DW, Zheng DL, Zheng L, Xing WJ, Zhou H, Cao DS, Zhang TC. NF-кB (p65) negatively regulates myocardin-induced cardiomyocyte hypertrophy through multiple mechanisms. Cellular signalling. 2014; 26:2738-2748. 